\title{
Ärztliche Arbeitsbedingungen im Krankenhaus
}

\author{
Ein Vergleich der Fachgebiete (iCept-Studie)
}

J. Bauer ${ }^{1}$, D. A. Groneberg ${ }^{1}$

Institute

1 Institut für Arbeits-, Sozial- und Umweltmedizin, Goethe-Universität, Frankfurt am Main

\author{
Korrespondenz \\ Jan Bauer \\ Institut für Arbeitsmedizin, \\ Sozialmedizin und Umwelt- \\ medizin \\ Goethe-Universität \\ Theodor-Stern-Kai 7 \\ 60590 Frankfurt am Main \\ Tel.: +49 69 6301-6650 \\ Fax: +49 $696301-7053$ \\ arbsozmed@med.uni- \\ frankfurt.de
}

Glossar
$95 \%-K I$
$95 \%-K o n f i d e n z i n t e r v a l l$
ER
Effort-Reward
ERI
Effort-Reward-
Imbalance
HNO
Hals-Nasen-Ohren
JDC
Job-Demand-Control
JDS
Job Diagnostic Survey
KFZA
Kurzfragebogen zur
Arbeitsplatzanalyse
MKG
Mund-Kiefer-Gesicht
OR
Quotenverhältnis
(Odds Ratio)
SD
Standardabweichung
(Standard Deviation)

\section{Zusammenfassung}

Hintergrund I Im Rahmen der iCept-Studie sind verschiedene ärztliche Berufsgruppen und ihre arbeitsplatzbezogenen Arbeitsbedingungen untersucht worden. Diese Studie stellt die verschiedenen in der iCept-Studie untersuchten Fachgebiete gegenüber.

Methoden I Die iCept-Studie stellt eine webbasierte Umfrage dar, an der insgesamt 7090 Krankenhausärzte teilnahmen. Die bisher veröffentlichen Daten im Rahmen der iCept-Studie werden in dieser Arbeit systematisch gegenübergestellt. Hierbei wird der Fokus auf Disstress und Berufszufriedenheit gelegt.

Ergebnisse I 53,9\% aller Befragten gaben ungünstige Arbeitsbedingungen im Sinne eines ER/JDCRatio $>1$ (Disstress) an. Vor allem chirurgische Fachgebiete wiesen hohe Disstress-Prävalenzen auf: Das Odds Ratio lag für die Chirurgie im Ver-

\section{Einleitung}

Stress am Arbeitsplatz kann viele Gründe und viele Auswirkungen haben. So hat ein negatives psychosoziales Arbeitsumfeld negative Auswirkung auf die mentale Gesundheit [1]. Darüber allerdings wann Stress genau entsteht, herrscht Unsicherheit, da bei der Stressentstehung die individuelle Verfassung der Arbeitnehmer eine sehr große Rolle spielt. Nichtsdestotrotz ist die Anzahl an möglichen definierten Auslösern sehr groß und diese werden regelmäßig in Studien bestätigt:

- Große Arbeitsbelastung,

- kleiner Handlungsspielraum oder

- lange Arbeitszeiten, um nur ein paar Beispiele zu nennen [2]. Gerade die Arbeitszeiten sind für die Gesundheit der Arbeitnehmer von großer Bedeutung [3].

Es sind zahlreiche allgemeine Modelle zur Stressentstehung beschrieben worden, wobei vor allem vier international anerkannt sind:

- Das Belastungs-Beanspruchungs-Modell von Rohmert \& Rutenfranz [4],

- das Job-Demand-Control-Modell von Karasek [5], gleich zur Anästhesiologie bei 1,59 (95\%-Konfidenzintervall $[1,35-1,86] \mathrm{p}<0,001)$. Patientenferne Fachgebiete wiesen im Vergleich dazu die geringsten Disstress-Prävalenzen auf: So lag das Odds Ratio für die Arbeitsmedizin im Vergleich zum Durchschnitt bei 0,37 ([0,19-0,71]; p<0,01). Weiter gaben 55,8\% aller Befragten an, sehr zufrieden mit ihrem Beruf zu sein.

Schlussfolgerung I Vor allem chirurgische Fachgebiete waren am häufigsten Disstress ausgesetzt. Da die Berufszufriedenheit bei fehlendem Disstress signifikant höher war, führt dies zur Schlussfolgerung, dass Zufriedenheit im Beruf und Stresserleben zwei abhängige Charakteristika einer sich wandelnden Arbeitskultur darstellen. Deswegen haben vor allem die chirurgischen Fachgebiete Nachholbedarf in Bezug auf eine Verbesserung der Arbeitsbedingungen, da somit die Stressbelastung gesenkt und damit die Zufriedenheit gesteigert werden kann.

- das transaktionale Stressmodell von Lazarus \& Folkman [6] und

- das Effort-Reward-Imbalance-Modell von Siegrist [7].

Allen Stressmodellen ist gemeinsam, dass sie Stressoren und Bewältigungsstrategien und damit ein Gleichgewicht beschreiben, welches bei "Stress“ in ein Ungleichgewicht zugunsten der Stressoren umschlägt. Allerdings ist Stress ein multifaktorielles Phänomen, weswegen keines der oben genannten Stressmodelle alle Aspekte der Stressentstehung abdecken kann. [8]

In unserer Studie wurden die Daten der iCept-Studie (iCept: Neologismus von engl. „i perception“ - Ich Wahrnehmung) ausgewertet. Ein Studienprotokoll [9] sowie verschiedene Publikationen sind im Rahmen der iCept-Studie bereits erschienen [10-13]. Die vorliegende Arbeit vergleicht die in der iCept-Studie untersuchten ärztlichen Fachgebiete. Hierbei wurden folgende Stressmodelle verwendet:

- Das Effort-Reward-Imbalance (ERI) Modell [7] und

- das Job-Demand-Control (JDC) Modell [5, 14].

Beide Stressmodelle sind gerade im medizinischen Arbeitsumfeld bereits mehrfach angewen- 
det worden und werden deswegen auch in dieser Studie bevorzugt verwendet.

Ein Blick auf die ärztliche Profession zeigt, dass die hierbei bestehenden Arbeitsbedingungen ein besonders hohes Stresspotenzial aufweisen [1517], auch mit den daraus folgenden Konsequenzen: So zeigte eine Befragung unter 790 Ärzten in Rheinland-Pfalz aus dem Jahr 2009 [18], dass jeder vierte Befragte (23\%) Anzeichen für eine berufsbedingte Depression hat.

Eine Untersuchung mit dem Fokus auf den verschiedenen Fachgebieten der ärztlichen Profession macht hier deswegen Sinn, da in den verschiedenen Fachgebieten unterschiedliche Arbeitsbedingungen vorliegen und damit auch ein unterschiedlich großes Stresspotenzial herrscht [19-21].

Abgesehen von den einzelnen Fachgebieten, scheinen speziell im Krankenhausbereich vier Bereiche ursächlich in der Entstehung von Stress zu sein: $\mathrm{Zu}$ den Problembereichen zählen die Arbeitsorganisation, Gratifikationskrisen, fehlender sozialer und fachlicher Rückhalt durch Kollegen/Vorgesetzte und individuelle fehlgeleitete Problemlösungsstrategien [22]. Vor allem auf die drei erstgenannten Punkte wurde in der iCept-Studie Bezug genommen.
Tab. 1 Odds Ratio (OR) und Mittelwertsdifferenzen $(\triangle)$ mit 95\%-KI, nach Fachgebiet (Referenz ist der Durchschnitt) ${ }^{*} p<0,05$, $* * \mathrm{p}<0,01$, $* * * p<0,001$

\begin{tabular}{|c|c|c|c|c|c|c|}
\hline & Effort & Reward & Job Demand & Control & $\begin{array}{l}\text { ER/JDC- } \\
\text { Ratio>1 }\end{array}$ & „sehr zufrieden“ \\
\hline & $\mathrm{M}(\mathrm{SD})$ & $\mathrm{M}(\mathrm{SD})$ & $\mathrm{M}(\mathrm{SD})$ & $\mathrm{M}(\mathrm{SD})$ & $\mathrm{n}(\%)$ & $\mathrm{n}(\%)$ \\
\hline \multirow{2}{*}{$\begin{array}{l}\text { Durchschnitt } \\
(n=7090)\end{array}$} & $15,2(2,7)$ & $13,6(2,6)$ & $8,3(1,6)$ & $6,9(1,8)$ & $3824(53,9)$ & $3956(55,8)$ \\
\hline & $\Delta[95 \%-\mathrm{KI}]$ & $\Delta[95 \%-\mathrm{KI}]$ & $\Delta[95 \%-\mathrm{KI}]$ & $\Delta[95 \%-\mathrm{KI}]$ & OR [95\%-KI] & OR [95\%-KI] \\
\hline $\begin{array}{l}\text { Allgemeinmedizin } \\
(n=66)\end{array}$ & $\begin{array}{l}-1,1[-1,8 \text { bis } \\
-0,5]^{* *}\end{array}$ & $\begin{array}{l}0,3[-0,3 \text { bis } \\
0,9]\end{array}$ & $\begin{array}{l}-0,2[-0,6 \text { bis } \\
0,2]\end{array}$ & $\begin{array}{l}0,6[0,2 \text { bis } \\
1,0]^{* *}\end{array}$ & $\begin{array}{l}0,67[0,41 \text { bis } \\
1,09]\end{array}$ & $\begin{array}{l}1,14[0,70 \text { bis } \\
1,87]\end{array}$ \\
\hline $\begin{array}{l}\text { Anästhesiologie } \\
(n=1321)\end{array}$ & $\begin{array}{l}-0,9[-1,0 \text { bis } \\
-0,7]^{* * *}\end{array}$ & $\begin{array}{l}0,3[0,1 \text { bis } \\
0,4]^{* *}\end{array}$ & $\begin{array}{l}-0,4[-0,5 \text { bis } \\
-0,3]^{* * *}\end{array}$ & $\begin{array}{l}-0,7[-0,8 \text { bis } \\
-0,6]^{* * *}\end{array}$ & $\begin{array}{l}0,76[0,67 \text { bis } \\
0,85]^{* * *}\end{array}$ & $\begin{array}{l}1,28[1,14 \text { bis } \\
1,45]^{* * *}\end{array}$ \\
\hline $\begin{array}{l}\text { Arbeitsmedizin } \\
(n=43)\end{array}$ & $\begin{array}{l}-0,2[-0,28 \text { bis } \\
-1,2]^{* * *}\end{array}$ & $\begin{array}{l}-0,5[-1,3 \text { bis } \\
0,3]\end{array}$ & $\begin{array}{l}-0,6[-1,1 \text { bis } \\
-0,2]^{* *}\end{array}$ & $\begin{array}{l}1,1[0,6 \text { bis } \\
1,7]^{* * *}\end{array}$ & $\begin{array}{l}0,37[0,19 \text { bis } \\
0,71]^{* *}\end{array}$ & $\begin{array}{l}1,48[0,79 \text { bis } \\
2,77]\end{array}$ \\
\hline $\begin{array}{l}\text { Augenheilkunde } \\
(n=59)\end{array}$ & $\begin{array}{l}0,2[-0,5 \text { bis } \\
0,9]\end{array}$ & $\begin{array}{l}0,1[-0,6 \text { bis } \\
0,8]\end{array}$ & $\begin{array}{l}0,2[-0,2 \text { bis } \\
0,7]\end{array}$ & $\begin{array}{l}0,2[-1,4 \text { bis } \\
-0,4]^{* * *}\end{array}$ & $\begin{array}{l}1,16[0,69 \text { bis } \\
1,95]\end{array}$ & $\begin{array}{l}0,93[0,56 \text { bis } \\
1,57]\end{array}$ \\
\hline $\begin{array}{l}\text { Chirurgie } \\
(n=1142)\end{array}$ & $\begin{array}{l}0,6[0,4 \text { bis } \\
0,8]^{* * *}\end{array}$ & $\begin{array}{l}-0,3[-0,4 \text { bis } \\
-0,1]^{* *}\end{array}$ & $\begin{array}{l}-0,2[-0,3 \text { bis } \\
-0,1]^{* *}\end{array}$ & $\begin{array}{l}-0,1[-0,3 \text { bis } \\
-0,0]^{*}\end{array}$ & $\begin{array}{l}1,20[1,06 \text { bis } \\
1,37]^{* *}\end{array}$ & $\begin{array}{l}0,87[0,76 \text { bis } \\
0,98]^{*}\end{array}$ \\
\hline $\begin{array}{l}\text { Gynäkologie } \\
(n=381)\end{array}$ & $\begin{array}{l}0,7[0,4 \text { bis } \\
0,9]^{* * *}\end{array}$ & $\begin{array}{l}-0,2[0,5 \text { bis } \\
0,0]\end{array}$ & $\begin{array}{l}0,1[-0,1 \text { bis } \\
0,3]\end{array}$ & $\begin{array}{l}-0,1[-0,3 \text { bis } \\
0,0]\end{array}$ & $\begin{array}{l}1,50[1,21 \text { bis } \\
1,86]^{* * *}\end{array}$ & $\begin{array}{l}0,97[0,79 \text { bis } \\
1,20]\end{array}$ \\
\hline HNO $(n=85)$ & $\begin{array}{l}0,8[0,2 \text { bis } \\
1,3]^{* *}\end{array}$ & $\begin{array}{l}-0,6[-1,2 \text { bis } \\
-0,0]^{*}\end{array}$ & $\begin{array}{l}0,2[-0,1 \text { bis } \\
0,6]\end{array}$ & $\begin{array}{l}-0,8[-1,2 \text { bis } \\
-0,4]^{* * *}\end{array}$ & $\begin{array}{l}2,60[1,59 \text { bis } \\
4,27]^{* * *}\end{array}$ & $\begin{array}{l}0,64[0,42 \text { bis } \\
0,99]^{*}\end{array}$ \\
\hline $\begin{array}{l}\text { Dermatologie } \\
(n=84)\end{array}$ & $\begin{array}{l}0,3[-0,3 \text { bis } \\
0,9]\end{array}$ & $\begin{array}{l}-0,7[-1,2 \text { bis } \\
-0,1]^{*}\end{array}$ & $\begin{array}{l}0,2[-0,1 \text { bis } \\
0,6]\end{array}$ & $\begin{array}{l}-0,2[-0,6 \text { bis } \\
0,2]\end{array}$ & $\begin{array}{l}1,54[0,98 \text { bis } \\
2,41]\end{array}$ & $\begin{array}{l}0,91[0,60 \text { bis } \\
1,41]\end{array}$ \\
\hline $\begin{array}{l}\text { Diagnost. Fach- } \\
\text { gebiete }(n=62)\end{array}$ & $\begin{array}{l}-1,2[-1,9 \text { bis } \\
-0,6]^{* * *}\end{array}$ & $\begin{array}{l}-0,1[-0,7 \text { bis } \\
0,6]\end{array}$ & $\begin{array}{l}-0,1[-0,5 \text { bis } \\
0,3]\end{array}$ & $0,8[0,4$ bis 1,3$]$ & $\begin{array}{l}0,41[0,24 \text { bis } \\
0,69]^{* *}\end{array}$ & $\begin{array}{l}1,34[0,80 \text { bis } \\
2,25]\end{array}$ \\
\hline $\begin{array}{l}\text { Innere Medizin } \\
(n=1696)\end{array}$ & $\begin{array}{l}0,7[0,6 \text { bis } \\
0,8]^{* * *}\end{array}$ & $\begin{array}{l}-0,2[-0,3 \text { bis } \\
-0,0]^{*}\end{array}$ & $\begin{array}{l}0,4[0,3 \text { bis } \\
0,5]^{* * *}\end{array}$ & $\begin{array}{l}0,2[0,1 \text { bis } \\
0,3]^{* * *}\end{array}$ & $\begin{array}{l}1,40[1,26 \text { bis } \\
1,56]^{* * *}\end{array}$ & $\begin{array}{l}0,74[0,66 \text { bis } \\
0,82]^{* * *}\end{array}$ \\
\hline Pädiatrie (n=455) & $\begin{array}{l}0,4[0,1 \text { bis } \\
0,6]^{* *}\end{array}$ & $\begin{array}{l}0,4[0,2 \text { bis } \\
0,7]^{* *}\end{array}$ & $\begin{array}{l}0,2[0,1 \text { bis } \\
0,4]^{* *}\end{array}$ & $\begin{array}{l}-0,1[-0,2 \text { bis } \\
0,1]\end{array}$ & $\begin{array}{l}1,18[0,97 \text { bis } \\
1,43]\end{array}$ & $\begin{array}{l}1,17[0,96 \text { bis } \\
1,42]\end{array}$ \\
\hline $\begin{array}{l}\text { Kinderpsychiatrie } \\
(n=78)\end{array}$ & $\begin{array}{l}-0,9[-1,5 \text { bis } \\
-0,3]^{* *}\end{array}$ & $\begin{array}{l}0,7[0,1 \text { bis } \\
1,3]^{*}\end{array}$ & $\begin{array}{l}0,1[-0,3 \text { bis } \\
0,4]\end{array}$ & $\begin{array}{l}1,4[1,0 \text { bis } \\
1,8]^{* * *}\end{array}$ & $\begin{array}{l}0,56[0,36 \text { bis } \\
0,89]^{*}\end{array}$ & $\begin{array}{l}1,20[0,76 \text { bis } \\
1,90]\end{array}$ \\
\hline $\begin{array}{l}\text { MKG-Chirurgie } \\
(n=28)\end{array}$ & $\begin{array}{l}1,8[0,8 \text { bis } \\
2,8]^{* * *}\end{array}$ & $\begin{array}{l}-1,5[-2,5 \text { bis } \\
-0,5]^{* *}\end{array}$ & $\begin{array}{l}0,2[-0,4 \text { bis } \\
0,8]\end{array}$ & $\begin{array}{l}-0,1[-0,8 \text { bis } \\
0,5]\end{array}$ & $\begin{array}{l}2,14[0,94 \text { bis } \\
4,85]\end{array}$ & $\begin{array}{l}0,59[0,28 \text { bis } \\
1,26]\end{array}$ \\
\hline $\begin{array}{l}\text { Neurochirurgie } \\
(n=78)\end{array}$ & $\begin{array}{l}-0,1[-0,7 \text { bis } \\
0,5]\end{array}$ & $\begin{array}{l}0,1[-0,5 \text { bis } \\
0,7]\end{array}$ & $\begin{array}{l}-0,4[-0,8 \text { bis } \\
-0,1]^{*}\end{array}$ & $\begin{array}{l}0,3[-0,1 \text { bis } \\
0,7]\end{array}$ & $\begin{array}{l}0,70[0,44 \text { bis } \\
1,09]\end{array}$ & $\begin{array}{l}1,78[1,1 \text { bis } \\
2,89]^{*}\end{array}$ \\
\hline $\begin{array}{l}\text { Neurologie } \\
(n=345)\end{array}$ & $\begin{array}{l}0,1[-0,2 \text { bis } \\
0,4]\end{array}$ & $\begin{array}{l}0,4[0,1 \text { bis } \\
0,7]^{* *}\end{array}$ & $\begin{array}{l}0,1[-0,1 \text { bis } \\
0,3]\end{array}$ & $\begin{array}{l}0,4[0,2 \text { bis } \\
0,6]^{* * *}\end{array}$ & $\begin{array}{l}0,92[0,75 \text { bis } \\
1,15]\end{array}$ & $\begin{array}{l}1,17[0,94 \text { bis } \\
1,45]\end{array}$ \\
\hline
\end{tabular}




\begin{tabular}{|c|c|c|c|c|c|c|}
\hline & Effort & Reward & Job Demand & Control & $\begin{array}{l}\text { ER/JDC- } \\
\text { Ratio>1 }\end{array}$ & „sehr zufrieden“ \\
\hline $\begin{array}{l}\text { Öffentl. Gesund- } \\
\text { heits, }(n=27)\end{array}$ & $\begin{array}{l}-1,6[-2,6 \text { bis } \\
-0,6]^{* *}\end{array}$ & $\begin{array}{l}-0,9[1,9 \text { bis } \\
0,1]\end{array}$ & $\begin{array}{l}-0,5[-1,1 \text { bis } \\
0,1]\end{array}$ & $\begin{array}{l}2,2[1,5 \text { bis } \\
2,9]^{* * *}\end{array}$ & $\begin{array}{l}0,24[0,10 \text { bis } \\
0,61]^{* *}\end{array}$ & $\begin{array}{l}1,88[0,82 \text { bis } \\
4,30]\end{array}$ \\
\hline $\begin{array}{l}\text { Phys./Reha. } \\
\text { Medizin }(n=85)\end{array}$ & $\begin{array}{l}-1,6[-2,2 \text { bis } \\
-1,0]^{* * *}\end{array}$ & $\begin{array}{l}-0,3[-0,9 \text { bis } \\
0,2]\end{array}$ & $\begin{array}{l}-0,6[-0,9 \text { bis } \\
-0,3]^{* *}\end{array}$ & $\begin{array}{l}0,2[-0,2 \text { bis } \\
0,6]\end{array}$ & $\begin{array}{l}0,63[0,41 \text { bis } \\
0,97]^{*}\end{array}$ & $\begin{array}{l}0,77[0,50 \text { bis } \\
1,19]\end{array}$ \\
\hline $\begin{array}{l}\text { Psychiatrie } \\
(n=508)\end{array}$ & $\begin{array}{l}-0,9[-1,2 \text { bis } \\
-0,7]^{* * *}\end{array}$ & $0,2[0,0$ bis 0,5$]$ & $\begin{array}{l}0,0[-0,2 \text { bis } \\
0,1]\end{array}$ & $\begin{array}{l}0,9[0,7 \text { bis } \\
1,0]^{* * *}\end{array}$ & $\begin{array}{l}0,56[0,46 \text { bis } \\
0,67]^{* * *}\end{array}$ & $\begin{array}{l}1,12[0,93 \text { bis } \\
1,34]\end{array}$ \\
\hline $\begin{array}{l}\text { Psychosomatik } \\
(n=126)\end{array}$ & $\begin{array}{l}-2,1[-2,6 \text { bis } \\
-1,6]^{* *}\end{array}$ & $\begin{array}{l}0,7[0,2 \text { bis } \\
1,1]^{* *}\end{array}$ & $\begin{array}{l}-0,2[-0,5 \text { bis } \\
0,0]\end{array}$ & $\begin{array}{l}1,1[0,7 \text { bis } \\
1,4]^{* * *}\end{array}$ & $\begin{array}{l}0,43[0,29 \text { bis } \\
0,62]^{* * *}\end{array}$ & $\begin{array}{l}1,84[1,25 \text { bis } \\
2,69]^{* *}\end{array}$ \\
\hline $\begin{array}{l}\text { Radiologie } \\
(n=208)\end{array}$ & $\begin{array}{l}-0,3[-0,6 \text { bis } \\
0,1]\end{array}$ & $\begin{array}{l}-0,1[-0,4 \text { bis } \\
0,3]\end{array}$ & $\begin{array}{l}0,1[-0,2 \text { bis } \\
0,3]\end{array}$ & $\begin{array}{l}-0,2[-0,5 \text { bis } \\
0,0]\end{array}$ & $\begin{array}{l}0,85[0,65 \text { bis } \\
1,13]\end{array}$ & $\begin{array}{l}1,19[0,90 \text { bis } \\
1,58]\end{array}$ \\
\hline $\begin{array}{l}\text { Strahlentherapie } \\
(n=32)\end{array}$ & $\begin{array}{l}-0,4[-1,3 \text { bis } \\
0,5]\end{array}$ & $\begin{array}{l}-0,7[-1,7 \text { bis } \\
0,2]\end{array}$ & $\begin{array}{l}-0,1[-0,7 \text { bis } \\
0,4]\end{array}$ & $\begin{array}{l}-0,5[-1,1 \text { bis } \\
0,2]\end{array}$ & $\begin{array}{l}1,25[0,61 \text { bis } \\
2,53]\end{array}$ & $\begin{array}{l}1,74[0,82 \text { bis } \\
3,69]\end{array}$ \\
\hline $\begin{array}{l}\text { Transfusionsmedi- } \\
\text { zin }(n=22)\end{array}$ & $\begin{array}{l}-0,9[-2,0 \text { bis } \\
0,2]\end{array}$ & $\begin{array}{l}-0,5[-1,6 \text { bis } \\
0,6]\end{array}$ & $\begin{array}{l}0,0[-0,7 \text { bis } \\
0,7]\end{array}$ & $\begin{array}{l}3,57[1,21 \text { bis } \\
10,54]^{*}\end{array}$ & $\begin{array}{l}0,71[0,31 \text { bis } \\
1,65]\end{array}$ & $\begin{array}{l}3,57[1,21 \text { bis } \\
10,54]^{*}\end{array}$ \\
\hline Urologie $(n=109)$ & $\begin{array}{l}0,4[-0,1 \text { bis } \\
0,9]\end{array}$ & $\begin{array}{l}0,1[-0,4 \text { bis } \\
0,6]\end{array}$ & $\begin{array}{l}-0,1[-0,4 \text { bis } \\
0,2]\end{array}$ & $\begin{array}{l}0,2[-0,1 \text { bis } \\
0,5]\end{array}$ & $\begin{array}{l}0,87[0,60 \text { bis } \\
1,27]\end{array}$ & $\begin{array}{l}1,09 \text { [0,74 bis } \\
1,59]\end{array}$ \\
\hline
\end{tabular}

Tab. 1 Fortsetzung

\section{Methoden}

Die Stichproberekrutierung erfolgte unter Mitgliedern des Marburger Bundes, welche mit einer standardisierten E-Mail einmalig angeschrieben wurden. Die Umfrage wurde mithilfe des webbasierten Online-Umfrage-Tools „2ask“ der amundis communications $\mathrm{GmbH}$ erstellt und durchgeführt.

Die Items des iCept-Fragebogens entstammen im Wesentlichen

- dem Kurz-Fragebogen zur Arbeitsanalyse (KFZA) von Prümper et al. [23],

- dem adaptierten Fragebogen nach Abele et al. [24] und

- der Kurzversion des Fragebogens zur EffortReward-Imbalance (ERI) von Siegrist et al. [25].

Die allgemeine Arbeitszufriedenheit wurde zusätzlich mit einem Item (JS1) der Skala „Allgemeine Arbeitszufriedenheit“ aus der deutschen Version des „Job Diagnostic Survey“ (JDS) von Schmidt et al. erfragt $[26,27]$.

Der verwendete Fragebogen wurde auf einer 4-stufigen Likert-Skala (stimme gar nicht zu, stimme nicht zu, stimme zu, stimme voll zu) beantwortet. Diesem Antwortformat wurde gegenüber der 5-stufigen Likert-Skala der Vorzug gegeben, da neuere Forschungsergebnisse zeigten, dass die 4-stufige Likert-Skala als Antwortformat höhere Antwortraten produziert, ohne dabei das Ergebnis zu verfälschen [25, 28].

Als Fachgebiete wurden die in der Musterberufsordnung der Bundesärztekammer (Stand: 2014) aufgeführten Fachgebiete definiert. Einem dieser
Fachgebiete mussten sich die Befragten selbst zuordnen. Hierauf beruht die Analyse der verschiedenen Fachgebiete. Ein Fokus der Studie liegt auf Disstress entsprechend der operationalisierten Definition der iCept-Studie: Die in der iCept-Studie erhobenen Skalenwerte für

- „effort" $\left(\mathrm{x}_{\mathrm{eff}}\right)$,

- ,job demand“ $\left(\mathrm{x}_{\mathrm{job}}\right)$,

- „reward“ $\left(\mathrm{x}_{\mathrm{rew}}\right)$ und

- "control“ ( $\left.\mathrm{x}_{\text {con }}\right)$

wurden in ein Verhältnis zueinander gebracht und dabei ein Kriterium (cut-off-Wert) festgelegt. Als cut-off-Wert für das Vorliegen von Disstress wurde ein JDC-Ratio > 1 und ein ER-Ratio > 1 definiert. Aufgrund variierender Anzahl der Items wurde zusätzlich jeweils ein Korrekturfaktor eingeführt $\left(c_{\text {eri }}=1,25\right.$ und $\left.c_{j d c}=0,75\right)$.

Es ist darauf hinzuweisen dass die „demand“Skala des JDC-Modell nach Karasek eine positive Korrelation mit der „effort“-Skala des ERI-Modells aufweist (zwischen $r=0,3$ und $r=0,6[29,30]$ ).

Darüber hinaus werden die Skalenwerte als selbständige Arbeitsplatzcharakteristika analysiert. In dieser Übersichtarbeit gelten dabei folgende methodische Grundsätze:

- Der persönliche Einsatz entspricht der Skala „effort“.

- Die wahrgenommene Belohnung entspricht der Skala „reward“.

- Die berufliche Anforderung entspricht der Skala ,job demands“.

- Der Handlungsspielraum entspricht der Skala „Control“.

Für die vergleichende statistische Datenanalyse der Fachgebiete wurde das Statistikprogramm 


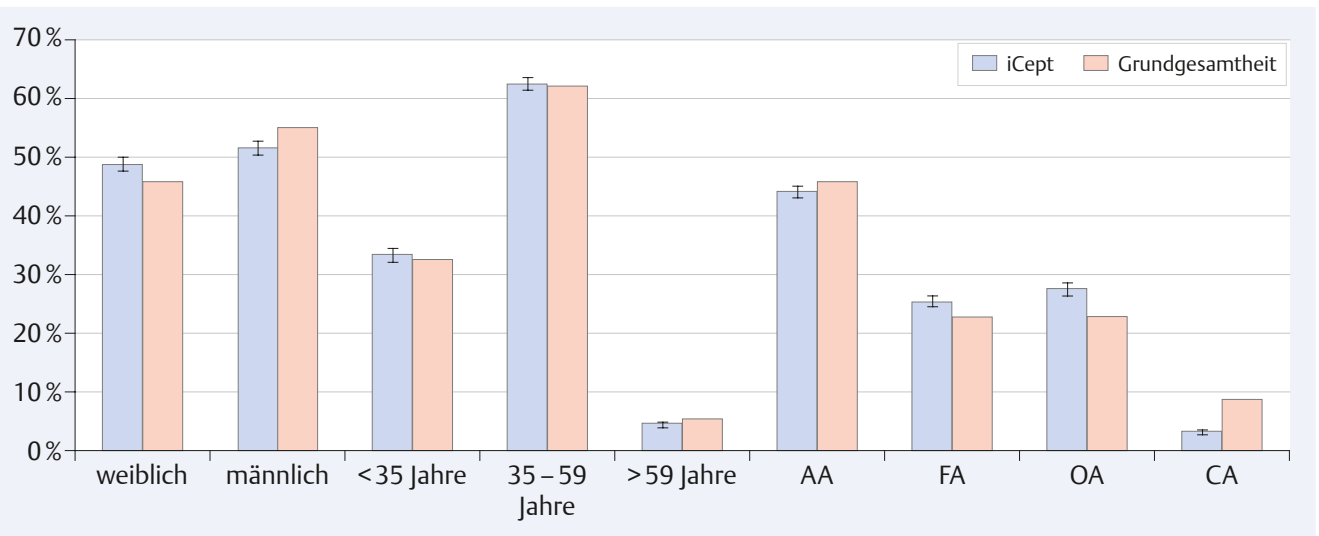

SPSS (Version 21) verwendet. Es wurden hierbei folgende statistische Testverfahren verwendet um auf signifikante Unterschiede zwischen einzelnen Fachgebieten zu testen: Es wurden das Quotenverhältnis (Odds Ratio) und die Mittelwertsunterschiede mithilfe des t-Tests und den dazugehörigen 95\%-Konfidenzintervallen berechnet.

\section{Ergebnisse}

Im Rahmen der gesamten iCept-Studie wurden insgesamt 39052 Krankenhausärzte/-ärztinnen in Deutschland per E-Mail angeschrieben. Von den 7181 abgegebenen Fragebögen waren 7090 vollständig ausgefüllt und konnten zur Auswertung herangezogen werden. Das entspricht einer Netto-Rücklaufquote von $18,2 \%$. Im Kontext der Grundgesamtheit (dazu zählen alle 169840 Krankenhausärzte in Deutschland im Jahr 2012) entspricht dies einem Anteil von 4,2\%. Die Datengrundlage für die Zahlen der Grundgesamtheit der Ärzte wird durch die Ärztestatistik der Bundesärztekammer [31] und das Statistische Bundesamt [32] gebildet.

In $>$ Abb. 1 sind die Kennzahlen der Grundgesamtheit derjenigen der Stichprobe gegenübergestellt. Wird die Verteilung der Geschlechter betrachtet, so zeigt sich eine Übereinstimmung mit der Verteilung in der Grundgesamtheit.

Das Durchschnittsalter der Stichprobe lag bei 40,4 Jahren (Bereich: 24-69 Jahre; SD: 9,4 Jahre). In der Grundgesamtheit lag dieses 2011 bei 41,1 Jahren [31].

Im Folgenden wird das Quotenverhältnis (Odds Ratio; OR) bzw. die Mittelwertsdifferenz $(\Delta)$, wenn nicht anders angegeben, immer im Verhältnis zum Gesamtdurchschnitt angegeben. Eine Gesamtübersicht über die vergleichende Betrachtung der Daten der iCept-Studie bezüglich der Fachgebiete ist aus Tab. 1 ersichtlich.

Der persönliche Einsatz („effort“) I Ein hoher Wert auf der effort-Skala entspricht einem hohen persönlichen Einsatz. Die beiden Fachgebiete mit den signifikant höchsten Skalenwerten waren die Hals-Nasen-Ohrenheilkunde (HNO) mit einer Mittelwertsdifferenz von $\Delta=0,8 \quad([0,2-1,3]$; $\mathrm{p}<0,01)$ und die Mund-Kiefer-Gesichtschirurgie (MKG) mit $\Delta=1,8([0,8-2,8] ; \mathrm{p}<0,001)$. Die beiden Fachgebiete mit den signifikant kleinsten Skalenwerten waren die Fachgebiete der physikalischen und rehabilitativen Medizin mit $\Delta=-1,6$ ([-2,2 bis $-1,0]$; $\mathrm{p}<0,001)$ und die Psychosomatik mit $\Delta=2,1$ $([-2,6$ bis $-1,6] ; \mathrm{p}<0,001)$.

Die wahrgenommene Belohnung („reward“) I Hohe Werte auf der reward-Skala deuten auf eine hohe wahrgenommene Belohnung hin. Hier waren die Fachgebiete mit den signifikant höchsten Skalenwerten die Kinder- und Jugendpsychiatrie und -psychotherapie mit $\Delta=0,7 \quad([0,1-1,3]$; $\mathrm{p}<0,05)$ und die Psychosomatik $\Delta=0,7([0,2-1,1]$; $\mathrm{p}<0,01)$. Die beiden Fachgebiete mit den signifikant kleinsten Skalenwerten waren die Dermatologie mit $\Delta=-0,7([-1,2$ bis $-0,1] ; \mathrm{p}<0,05)$ und die MKG mit $\Delta=-1,5$ ([-2,5 bis $-0,5] ; \mathrm{p}<0,01)$.

Die beruflichen Anforderungen (,job demands“) | Große Berufliche Anforderungen drückten sich durch hohe Werte auf der job-demands-Skala aus. Das Fachgebiet, welches den höchsten Skalenwert aufwies, war das Fachgebiet der Inneren Medizin mit $\Delta=0,4([0,2-0,7] ; \mathrm{p}<0,001)$. Als relativ niedrig sind die beruflichen Anforderungen

- in der Anästhesiologie mit $\Delta=-0,4$ ([-0,5 bis $-0,3] ; p<0,001)$,

- der Arbeitsmedizin mit $\Delta=-0,6$ ([-1,1 bis $-0,2]$; $\mathrm{p}<0,01$ ) und

- dem Fachgebiet der physikalischen und rehabilitativen Medizin mit ebenfalls $\Delta=-0,6$ ([-0,9 bis - 0,3 ]; $p<0,01$ )

wahrgenommen worden.

Der Handlungsspielraum („control“) I Das Fachgebiet der Kinderpsychiatrie wies einen besonders großen Handlungsspielraum auf (hoher Wert auf der control-Skala) mit $\Delta=1,4$ ([1,0-1,8]; $\mathrm{p}<0,001)$. Als signifikant niedriger ist der Handlungsspielraum in der Anästhesiologie mit $\Delta=-0,7$ $([0,8-0,6] ; \mathrm{p}<0,001)$ wahrgenommen worden.
Abb. 1 Kennzahlen von Stichprobe und Grundgesamtheit;

AA: Assistenzärzte / -ärztinnen,

FA: Fachärzte / -ärztinnen, OA: Oberärzte/-ärztinnen, CA: Chefärzte/-ärztinnen Fehlerbalken: $95 \%-\mathrm{KI}$ 


$$
\begin{array}{r}
\text { HNO }(n=85) \\
\text { MKG-Chirurgie }(n=28) \\
\text { Dermatologie }(n=84) \\
\text { Gynäkologie }(n=381) \\
\text { Innere Medizin }(n=1696) \\
\text { Strahlentherapie }(n=32) \\
\text { Chirurgie }(n=1142) \\
\text { Pädiatrie }(n=455) \\
\text { Augenheilkunde }(n=59) \\
\text { Neurologie }(n=345) \\
\text { Urologie }(n=109) \\
\text { Radiologie }(n=208) \\
\text { Anästhesiologie }(n=1321) \\
\text { Transfusionsmedizin }(n=22) \\
\text { Neurochirurgie }(n=78) \\
\text { Allgemeinmedizin }(n=66) \\
\text { Phys./Reha. Medizin }(n=85) \\
\text { Psychiatrie }(n=508) \\
\text { Kinderpsychiatrie }(n=78) \\
\text { Psychosomatik }(n=126)
\end{array}
$$$$
0,1
$$

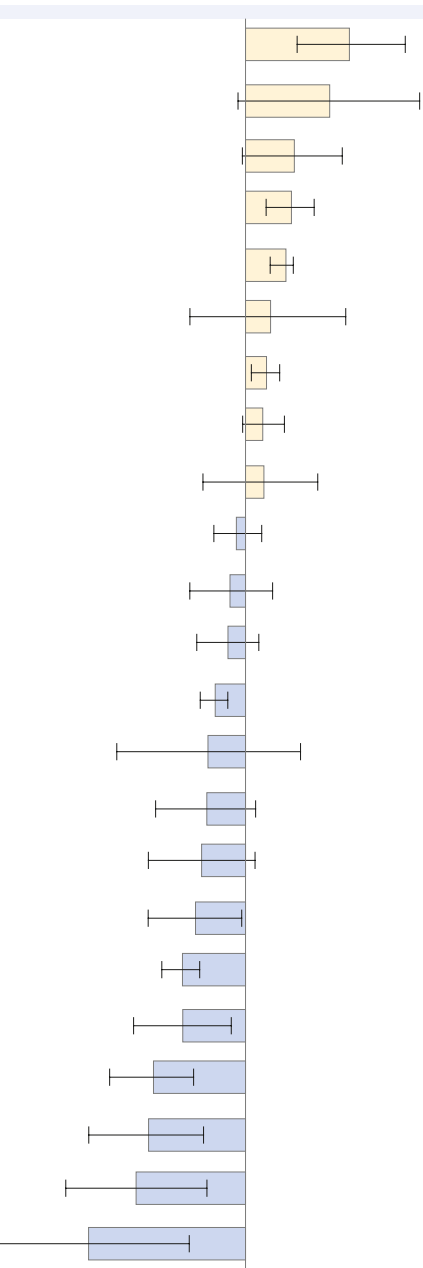

0,1
- HNO,

- MKG,

- Gynäkologie und

- Dermatologie.

Die höchste Prävalenz wies das Fachgebiet der HNO mit einem Quotenverhältnis von 2,60 auf $([1,59-4,27] ; \mathrm{p}<0,001)$. So lag beispielsweise auch das Quotenverhältnis der Disstress-Prävalenzen der Chirurgie zur Anästhesiologie bei 1,59 ([1,3-1,86]).

Disstress ist ein weit verbreitetes Arbeitsplatzmerkmal innerhalb medizinischer Fachgebiete. Abgesehen von der Neurochirurgie und der Urologie scheint in operativen Fachgebieten vergleichsweise häufig Disstress erlebt zu werden.

Die Berufszufriedenheit (JS1) I In der iCept-Studie gaben 55,8\% und damit mehr als jeder zweite Befragte an, sehr zufrieden mit seinem Beruf zu sein. Allerdings gab es in Bezug auf die Berufszufriedenheit deutliche Unterschiede zwischen den Fachgebieten, welche in $\mathbf{A b b}$. $\mathbf{3}$ dargestellt sind.

Der Anteil der Befragten, die angaben sehr zufrieden mit ihrem Beruf zu sein, war überdurchschnittlich hoch in folgenden Fachgebieten:

- Transfusionsmedizin: OR=3,57 ([1,21-10,54]; $\mathrm{p}<0,05)$

- Psychosomatik: OR=1,84([1,25-2,69]; $\mathrm{p}<0,01)$

- Neurochirurgie: OR=1,78 ([1,1-2,89]; $\mathrm{p}<0,05)$

- Anästhesiologie: $\mathrm{OR}=1,28([1,14-1,45] ; \mathrm{p}<0,001)$. Entsprechend der oben dargestellten hohen Disstress-Prävalenz in der HNO war der Anteil der Befragten Ärzte und Ärztinnen, die angaben sehr zufrieden mit ihrem Beruf zu sein, hier mit $\mathrm{OR}=0,64([0,42-0,99] ; \mathrm{p}<0,05)$ auch signifikant niedriger. Auch gaben mit $\mathrm{OR}=0,74([0,66-0,82]$; $\mathrm{p}<0,001)$ signifikant weniger Befragte in der Inneren Medizin an, sehr zufrieden mit ihrem Beruf zu sein.

Das „Risiko“ sehr zufrieden mit seinem Beruf zu sein, war damit unter den Befragten welche keinen Disstress aufwiesen 7,89 mal höher ([7,078,80]; $\mathrm{p}<0,001$ ) als unter denjenigen Befragten, welche Disstress erlebten.

Eine weitergehende Analyse zeigt, dass unter zwei Fachgebieten mit den geringsten Prävalenzen für Disstress ausschließlich patientenferne Gebiete zu finden waren:

- Zusammengefasste diagnostische Fachgebiete (Mikrobiologie/Virologie, Laboratoriumsmedizin und die Pathologie): OR=0,41 ([0,240,69]; $\mathrm{p}<0,001$ )

- Die Arbeitsmedizin: OR=0,37 ([0,19-0,71]; $\mathrm{p}<0,01$ )

Umgekehrt waren unter den vier Fachgebieten mit den höchsten Prävalenzen für Disstress ausschließlich chirurgische Gebiete zu finden:

\section{Diskussion}

Die Tatsache, dass mehr als jeder zweite Befragte ungünstige Arbeitsbedingungen wahrnahm, lässt die ärztliche Arbeitsumgebung „Krankenhaus“ als relativ stressbehaftet erscheinen. In der vergleichenden Betrachtung der Ergebnisse der iCept-Studie sind darüber hinaus die patientenfernen Fachgebiete mit geringeren DisstressPrävalenzen auffällig. Daraus könnte geschlossen werden, dass der Patientenkontakt zu einem 
höheren Stresspotenzial der Arbeitsbedingungen führt. Der Aspekt des Patientenkontaktes wurde in dieser Studie allerdings nicht direkt untersucht und somit kann auf Zusammenhänge nur indirekt geschlossen werden. Der Befund, dass vergleichsweise viele operative Fachgebiete mit hohen Disstress-Prävalenzen einhergehen, erscheint plausibel. Allerdings wies eine vergleichbare Studie [33] unter 1311 deutschen Klinikchirurgen / -chirurginnen Disstress-Prävalenzen von $22,4 \%$ bzw. $25,1 \%$ (ER/JDC-Ratio > 1) aus. In der vorliegenden Studie liegt diese bei 58,5\% und damit mehr als doppelt so hoch.

Wird der Zusammenhang von Arbeitsplatzmerkmalen (Disstress) mit den psychischen Folgen (wie z.B. Burnout) betrachtet, so ergeben sich Übereinstimmungen. Allerdings dürfen beide Parameter nicht gleichgesetzt werden, da es sich um unterschiedliche Merkmalsbereiche handelt. Eine große Untersuchung unter 7288 amerikanischen Ärztinnen und Ärzten [34] zeigte vor allem für die Fachgebiete mit Patientenerstkontakt eine hohe Burnout-Rate. Durchschnittlich berichteten $45,8 \%$ der Befragten über Burnout-Symptome. Ein Vergleich dieser und der iCept-Studie ist in - Abb. 4 dargestellt. Es ist allerdings anzumerken, dass die Autoren der genannten Studie BurnoutPrävalenz berichteten und in der iCept-Studie ausschließlich Disstress-Prävalenzen angegeben wurden. Deswegen können Schlüsse aus dem direkten Vergleich nur mit großer Vorsicht gezogen werden. Unter den genannten Limitationen ergeben sich hier Übereinstimmungen zwischen Disstress und Burnout.

In einer weiteren Studie unter 314 italienischen Radiologen [35] zeigten $28 \%$ ein ER-Ratio $>1$ und $24 \%$ ein JDC-Ratio > 1 . In der vorliegenden Studie war dies bei $59 \%$ bzw. 72\% der Befragten Radiologen der Fall.

Im Vergleich mit anderen akademischen Berufen zeigen sich auch hier Unterschiede: Laut einer Studie unter 949 Lehrern/innen in Deutschland liegt die Quote der Befragten mit Anzeichen für Disstress bei 21,6\% (ER-Ratio>1) [36]. Bei der vorliegenden Studie gaben dies 63,4\% an.

Eine Studie aus dem Jahr 2013 [37], in der Daten verschiedener Berufsgruppen aus der Gutenberg Health Study aus dem Jahr 2008 untersucht worden sind, wies für Ärzte und Pharmazeuten einen Skalenwert für „effort“ mit 14,8 (SD: 3,6) aus. Journalistische Fachgebiete kamen auf einen Skalenwert von 11,9 (SD: 2,8). In der iCept-Studie lag der durchschnittliche Skalenwert für „effort“ bei 15,2 (SD: 2,7). Damit erscheint der Skalenwert der iCept-Studie ebenfalls plausibel, wenn auch auf einem vergleichsweise hohen Niveau.

Wird die Berufszufriedenheit innerhalb der iCeptStudie betrachtet, so erscheint der Durchschnitt durchaus zufrieden mit seinem Beruf zu sein. Der Vergleich mit einer aktuellen Studie der HansBöckler Stiftung [38] bestätigt diesen Befund: Hier hatten Ärzte durchschnittlich den höchsten Zufriedenheitswert im Vergleich mit anderen Beschäftigen. Des Weiteren gibt diese Studie einen Ausblick auf die Zukunftserwartung der Ärzte: So gaben 61,5\% der Befragten an, sie gehen davon aus, dass die berufliche Verantwortung zunehmen werde. $63,5 \%$ gehen nicht davon aus, dass hierbei die Handlungsspielräume zunehmen werden. Im Sinne der genannten Stresstheorien (ER/JDC) bleibt somit fraglich, ob die derzeitige Situation in Zukunft verbessert wird.

55,8\% der Befragten gaben an sehr zufrieden mit ihrem Beruf zu sein. Dieser Anteil scheint im Vergleich mit anderen Untersuchungen plausibel: In einer Studie unter chinesischen Beschäftigen in der Primärversorgung [39] waren 47,6\% zufrieden oder sehr zufrieden mit ihrem Beruf. Unter Radiologen waren in einer amerikanischen Studie aus dem Jahr 2013 [40] 53\% der Befragten sehr zufrieden mit ihrem Beruf. Bei den Radiologen der iCept-Studie gaben dies 60,1\% an.

Abb. 3 Odds Ratio der Anteile an Befragten mit hoher Berufszufriedenheit, nach Fachgebieten

Transfusionsmedizin ( $\mathrm{n}=22)$

Öffentl. Gesundheitswesen ( $n=27)$

Psychosomatik $(n=126)$

Neurochirurgie $(n=78)$

Strahlentherapie $(n=32)$

Arbeitsmedizin $(n=43)$

Diagnost. Fachgebiete $(n=62)$

Anästhesiologie $(n=1321)$

Kinderpsychiatrie $(\mathrm{n}=78)$

Radiologie $(n=208)$

Neurologie $(n=345)$

Pädiatrie $(n=455)$

Allgemeinmedizin $(n=66)$

Psychiatrie $(n=508)$

Urologie $(n=109)$

Gynäkologie $(n=381)$

Augenheilkunde ( $n=59)$

Dermatologie $(n=84)$

Chirurgie $(n=1142)$

Phys./Reha. Medizin ( $n=85$ )

Innere Medizin ( $n=1696)$

HNO $(n=85)$

MKG-Chirurgie $(n=28)$ 
Limitationen

Die Repräsentativität der iCept-Studie muss kritisch gesehen werden: Zunächst einmal zeigt - Abb. 1 eine relativ hohe Übereinstimmung in wichtigen Merkmalen mit der Grundgesamtheit. Dies könnte auf eine gegebene Repräsentativität hindeuten. Andererseits war die Rücklaufquote mit $18,2 \%$ relativ gering, gerade auch im Vergleich mit anderen vergleichbaren Studien [41]. Dadurch kann trotz der hohen absoluten Teilnehmerzahl eine Antwortverzerrung durch ein Selektionsbias bzw. durch die „Non-Responder“ nicht gänzlich ausgeschlossen werden. Prinzipiell scheint es wahrscheinlich, dass durch das Selektionsbias die eher unzufriedenen Ärzte an dieser Studie teilgenommen haben. Dies muss sicherlich als Limitation betrachtet werden und könnte die Überzeichnung der Disstress-Prävalenzen im Vergleich mit den oben beschriebenen Studien erklären.

Prinzipiell muss auch darauf hingewiesen werden, dass alle Schlussfolgerungen auf subjektiven Aussagen und Einschätzungen der Befragten beruhen und damit keine absoluten Aussagen getroffen werden können. Auch die multiple Testung der Fächerunterschiede für die jeweiligen Outcomes beinhaltet das Risiko falsch positiver Unterschiede und muss deshalb als limitierender Faktor betrachtet werden. Ebenso besteht bei der vergleichenden Auswertung der Disstress-Prävalenzen der limitierende Faktor der ungleichen Stichprobengrößen der Fachgebiete. Dies führt zu einer Überbetonung der Fachgebiete mit großen Stichprobenzahlen mit den daraus folgenden Risiken bei der Signifikanzbewertung.
Für den Vergleich dieser Studie mit anderen nationalen und internationalen Studien müssen weitere diverse Limitationen beachtet werden: Einerseits stellt die vorliegende Studie eine Bestandsaufnahme in einem dynamischen Arbeitsumfeld dar, wodurch die zeitliche Latenz zwischen Studien beachtet werden muss. Darüber hinaus sind gerade bei internationalen Vergleichen nationale Besonderheiten zu beachten, welche Auswirkung auf Arbeitsplatzmerkmale haben können. Andererseits müssen methodische Unterschiede zwischen einzelnen Studien beachtet werden, was zu Verzerrungen führen könnte.

\section{Konsequenz für Klinik und Praxis}

- Um die Arbeitnehmer dauerhaft leistungsfähig und psychisch gesund zu halten, müssen Arbeitsbedingungen verbessert werden.

- Hierbei gilt es ein Gleichgewicht zu finden zwischen Belastung und Anforderung.

- Konkrete Maßnahmen beinhalten

> die Selbstbestimmung der Arbeitnehmer zu erhöhen sowie

> die Handlungsspielräume zu vergrößern.

- Eine persönliche Verausgabungstendenz sollte sowohl von Arbeitnehmer-, als auch Arbeitgeberseite kritisch hinterfragt werden.

- Regelmäßige Mitarbeitergespräche können helfen, Situationen mit erhöhtem Disstresspotenzial zu identifizieren und zu vermeiden.
Abb. 4 Prävalenzen von Burnout [34] und Disstress (iCept), nach Fachgebiet.

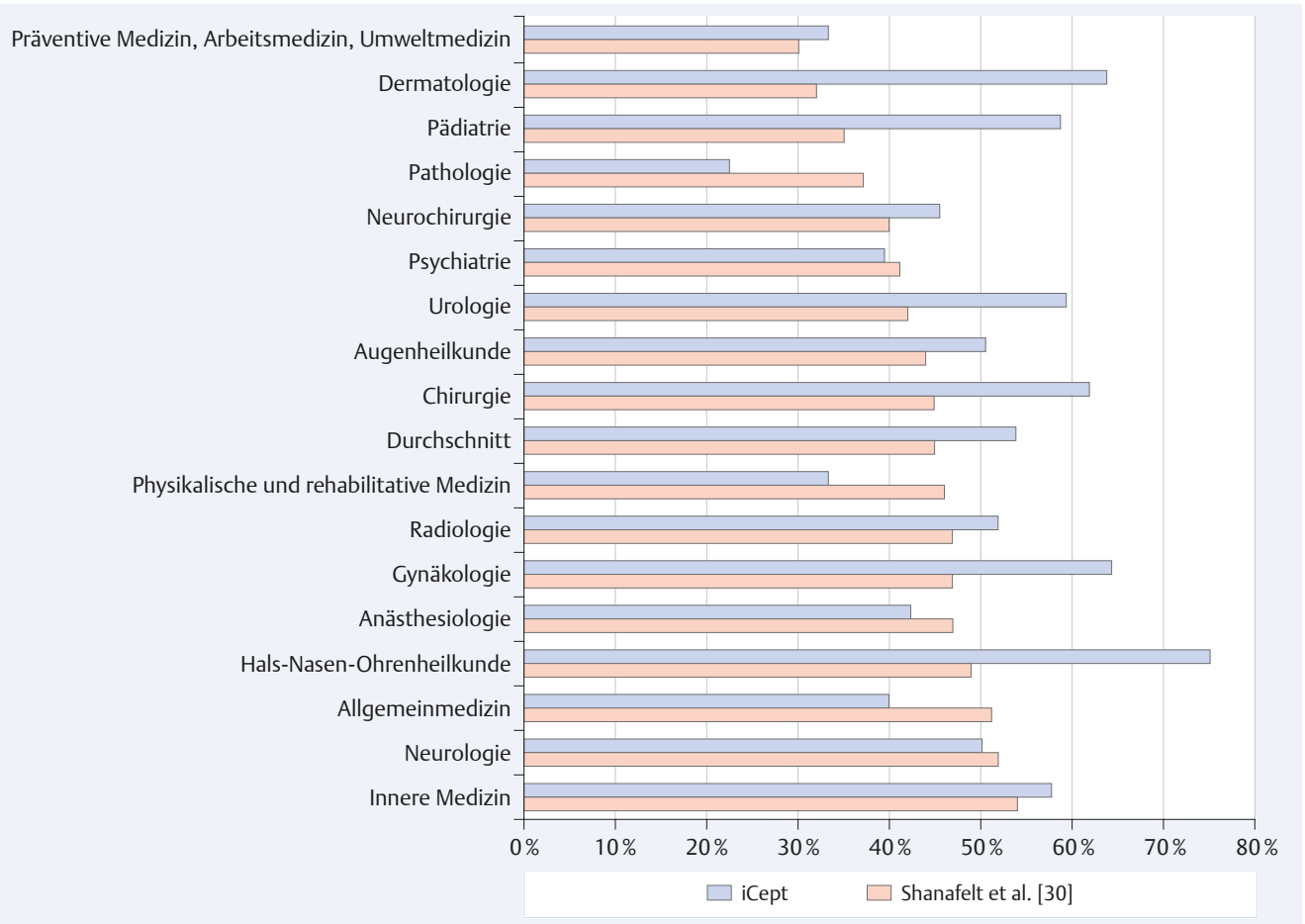




\section{Danksagungen}

Wir danken Herrn Prof. Dr. med. Frank Ulrich Montgomery. Diese Studie wurde unterstützt durch den „Marburger Bund” und die „Bundesärztekammer".

\section{Literatur}

1 Nolfe G, Mancini P, Mancusi R et al. Work-related psychopathology: rates in different work activities and relationship between subjective perception of work distress and psychiatric disturbances. Work 2014; 47: 501-508

2 Sugiura-Ogasawara M, Suzuki S, Kitazawa M et al. Career satisfaction level, mental distress, and gender differences in working conditions among Japanese obstetricians and gynecologists. J Obstet Gynaecol Res 2012; 38: 550-558

3 Artazcoz L, Cortes I, Escriba-Agueir V et al. Long working hours and health status among employees in europe: Between-country differences. Scand J Work Env Heal 2013; 39: 369-378

4 Rohmert W, Rutenfranz J. Arbeitswissenschaftliche Beurteilung der Belastung und Beanspruchung an unterschiedlichen Industriearbeitsplätzen. Bonn: Bundesministerium für Arbeit und Sozialordnung; 1975

5 Karasek RA. Job demands, job decision latitude and mental strain: Implications for job redesign. Adm Sci Q 1979; 24: 285-308

6 Lazarus RS, Folkman S. Stress, appraisal and coping. New York: Springer; 1984

7 Siegrist J. Adverse health effects of high-effort / low-reward conditions. J Occup Heal Psychol 1996; 1: 27-41

8 Bamberg E, Keller M, Wohlert C, Zeh A. BGWStresskonzept: Das arbeitspsychologische Stressmodell. Hamburg: Berufsgenossenschaft für Gesundheitsdienst und Wohlfahrtspflege (BGW); 2006

9 Bauer J, Groneberg DA. Perception of stress-related working conditions in hospitals (iCept-study): a comparison between physicians and medical students. J Occup Med Toxicol 2013; 8: 3

10 Bauer J, Groneberg DA. Disstress und Berufszufriedenheit unter Klinikärzten der Inneren Medizin . Internist (Berl) 2014; 55: 1242-1250

11 Bauer J, Groneberg DA. Disstress in der Chirurgie - Eine Untersuchung in deutschen Krankenhäusern. Zentralbl Chir 2014 DOI: $10.1055 / \mathrm{s}-0033-1350893$

12 Bauer J, Groneberg DA. Stress und Berufszufriedenheit im Fachgebiet der stationären Anästhesiologie. Anaesthesist 2014; 63: 32-40

13 Bauer J, Groneberg DA. Ärztlicher Disstress - eine Untersuchung baden-württembergischer Ärztinnen und Ärzte in Krankenhäusern. Dtsch Med Wochenschr 2013; 138: 2401-2406

14 Karasek RA, Theorell T. Healthy work: stress, productivity and the reconstruction of working life. New York: New York Basic Books, 1990

15 Cohen JS, Patten S. Well-being in residency training: a survey examining resident physician satisfaction both within and outside of residency training and mental health in Alberta. BMC Med Educ 2005; 5: 21

16 Katz JT, Lilly CM, Stone PH et al. Effect of reducing interns' work hours on serious medical errors in intensive care units. N Engl J med 2004; 351: 1838-1848

17 Baldwin DC, Daugherty SR, Tsai R, Scotti MJ. A national survey of residents' self-reported work hours: Thinking beyond specialty. Acad Med 2003; 78: $1154-1163$
18 Unrath M, Zeeb H, Letzel S et al. The mental health of primary care physicians in Rhineland-Palatinate, Germany: the prevalence of problems and identification of possible risk factors. Dtsch Arztebl Int 2012; 109: 201-207

19 Michalsen A, Hillert A. Burn-out in Anästhesie und Intensivmedizin. Teil 1.: Klärung und kritische Wertung des Begriffs. Anaesthesist 2011; 60: 23-30

20 Böhmert M, Kuhnert S, Nienhaus A. Psychologica stress and strain in dialysis staff - a systematic review. J Ren Care 2011; 49: 178-189

21 Pereira SM, Fonseca AM, Carvalho AS. Burnout in palliative care: a systematic review. Nurs Ethics 2011; 18: 317-326

22 Angerer P, Petru R, Nowak D, Weigl M. Arbeitsbedingungen und Depression bei Ärzten. Dtsch Med Wochenschr 2008; 133: 26-29

23 Prümper J, Hartmannsgruber K, Frese M. KFZA. Kurz-Fragebogen zur Arbeitsanalyse. Zeitschrift für Arbeits- und Organ 1995; 39: 125-132

24 Abele AE, Stief M, Heismann C, Spurk D. Berufliche Laufbahnentwicklung von Akademikerinnen und Akademikern der Universität Erlangen-Nürnberg (BELA-E): Dritte Erhebung der prospektiven Längsschnittstudie Fragebogen und Grundauswertung. Erlangen: Friedrich-Alexander-Universität Erlangen-Nürnberg; 2006

25 Siegrist J, Starke D, Chandola T et al. The measurement of effort-reward imbalance at work: European comparisons. Soc Sci Med 2004; 58: 1483-1499

26 Schmidt K-H, Kleinbeck U. Job Diagnostic Survey (JDS - deutsche Fassung). In: Ulich E (ed.). Dunkel H.: Handbuch psychologischer Arbeitsanalyseverfahren. Schriftenreihe Mensch-Technik-Organisation. Zürich: vdf Hochschulverlag AG; 1999: 205-230

27 Hackman JR, Oldham GR. Development of the Job Diagnostic Survey. J Appl Psychol 1975; 60: 159-170

28 Siegrist J, Wege N, Pühlhofer F, Wahrendorf M. A short generic measure of work stress in the era of globalization: effort-reward imbalance. Int Arch Occup Env Heal 2009; 82: 1005-1013

29 Calnan M, Wainwright D, Almond S. Job Strain, Effort Reward and Mental Distress: A Study of General Practice. Work Stress 2001; 14: 297-311

30 Tsutsumi A, Kayaba K, Theorell T, Siegrist ]. Association between job stress and depression among Japanese employees threatened by job loss in comparison between two complementary job-stress models. Scand J Work Env Heal 2001; 27: $146-153$

31 Bundesärztekammer und Kassenärztliche Bundesvereinigung. Ärztestatistik. 2013;

32 Statistisches Bundesamt. Grunddaten der Krankenhäuser. Fachserie 12 R 611 2012; 1-137

33 Klein J, Grosse Frie K, Blum K, von dem Knesebeck O. Psychosocial stress at work and perceived quality of care among clinicians in surgery. BMC Heal Serv Res 2011; 11: 109

34 Shanafelt TD, Boone S, Tan L et al. Burnout and satisfaction with work-life balance among US physicians relative to the general US population. Arch Intern Med 2012; 172: 1377-1385

35 Magnavita N, Fileni A, Magnavita G et al. Work stress in radiologists. A pilot study. Radiol Med 2008; 113: 329-346

36 Unterbrink T, Hack A, Pfeifer R et al. Burnout and effort-reward-imbalance in a sample of 949 German teachers. Int Arch Occup Env Heal 2007; 80: 433-441 
37 Nuebling M, Seidler A, Garthus-Niegel S et al. The Gutenberg Health Study: measuring psychosocial factors at work and predicting health and work-related outcomes with the ERI and the COPSOQ questionnaire. BMC Public Health 2013; 13: 538

38 Bräutigam C, Evans M, Hilbert J, Öz F. Arbeitsreport Krankenhaus - Eine Online-Befragung von Beschäftigten deutscher Krankenhäuser (Arbeitspapier Nr. 306). Düsseldorf: Hans Böckler Stiftung; 2014
39 Shi L, Song K, Rane S et al. Factors associated with job satisfaction by Chinese primary care providers. Prim Heal Care Res Dev 2014; 15: 46-57

40 Ruchman RB, Kwak AJ, Jaeger J, Sayegh A. Job satisfaction of program directors in radiology: a survey of current program directors. AJR Am J Roentgenol 2013; 200: 238-247

41 Cummings SM, Savitz LA, Konrad TR. Reported Response Rates to Mailed Physician Questionnaires. Heal Serv Res 2001; 35: 1347-1355

Interessenkonflikt

Die Autoren geben an, dass kein Interessenkonflikt besteht.

DOI 10.1055/s-0041-103165

Dtsch Med Wochenschr 2015; 140: e150-e158

(c) Georg Thieme Verlag KG . Stuttgart · New York .

ISSN 0012-0472 